\title{
Energetics of $\mathrm{H}$ and $\mathrm{NH}_{2}$ on $\mathrm{GaN}(10 \overline{10})$ and implications for the origin of nanopipe defects
}

\author{
John E. Northrup and R. Di Felice \\ Xerox Palo Alto Research Center, 3333 Coyote Hill Road, Palo Alto, California 94304 \\ Jörg Neugebauer \\ Fritz-Haber-Institut der Max-Planck-Gesellschaft, Faradayweg 4-6, D-14195 Berlin, Germany \\ (Received 7 April 1997)
}

\begin{abstract}
We present first-principles calculations of the formation energy for H-terminated $\mathrm{GaN}(10 \overline{1} 0)$ surfaces. The calculations indicate that $\mathrm{H}$ adsorption on $\mathrm{GaN}(10 \overline{10})$ will proceed by saturation of pairs of $\mathrm{Ga}$ and $\mathrm{N}$ dangling bonds rather than through exclusive occupation of only one type of bonding site. At $T=0$, the surface energy of the fully H-terminated surface is found to be $0.02 \mathrm{eV} /$ cell, compared to $1.95 \mathrm{eV} /$ cell for the bare surface. We present results for the N-H and Ga-H stretching and bending eigenfrequencies. Dissociative adsorption of $\mathrm{NH}_{3}$ via the formation of $\mathrm{N}-\mathrm{H}$ and $\mathrm{Ga}-\mathrm{NH}_{2}$ bonds is exothermic and reduces the surface formation energy to a value which is less than $0.1 \mathrm{eV}$ at $T=0$. The implications of these results for the origin of nanopipe defects in $\mathrm{GaN}$ are examined. [S0163-1829(97)51832-7]
\end{abstract}

The most commonly used technique for the growth of $\mathrm{GaN}$ epitaxial films is vapor phase epitaxy in which molecules such as $\mathrm{H}_{2}, \mathrm{NH}_{3}$, and $\mathrm{Ga}\left(\mathrm{CH}_{3}\right)_{3}$ flow into a reaction chamber and interact with a heated substrate. The substrate temperature is $\sim 1300 \mathrm{~K}$ and the typical pressure in the reactor is between 0.1 and $1 \mathrm{~atm}$. Under these conditions the GaN surface interacts with a H-rich ambient, and it is therefore of interest to examine the energetics of $\mathrm{H}$ and $\mathrm{NH}_{2}$ on $\mathrm{GaN}$ surfaces. In this work we present first-principles calculations of the formation energy of the $\mathrm{GaN}(1010)$ surface with $\mathrm{H}$ saturating the $\mathrm{Ga}$ and $\mathrm{N}$ dangling bonds.

Part of the motivation for our choice of the (1010) surface stems from the fact that this surface is the bounding internal facet exhibited by hollow-core screw dislocations ${ }^{1}$ in $\mathrm{GaN}$. Voids associated with [0001] screw dislocations have been observed in epitaxially grown films of GaN by Qian et al. ${ }^{2}$ and recent work by Cherns et $a l_{.}^{3}$ and Romano ${ }^{4}$ have confirmed these results. These defects typically have a diameter of between 3 and $25 \mathrm{~nm} ;{ }^{2-4}$ hence they have been termed nanopipes. ${ }^{2}$ The nanopipe defects exhibit (1010) faceted walls, and appear to extend throughout the entire thickness of the epitaxial film. Since nanopipes are present in some epitaxial films ${ }^{3}$ with concentrations as large as $10^{8} / \mathrm{cm}^{2}$ there is considerable interest in understanding their origin.

Frank argued that a screw dislocation of a sufficiently large Burgers vector would exhibit an equilibrium structure in which the core of the dislocation is an empty tube. ${ }^{1}$ Removal of the strained material near the dislocation core will be exothermic provided the energy cost of creating the internal surfaces of the void is offset by the reduction in strain energy. Within Frank's equilibrium theory, the surface energy, the shear modulus of rigidity, and the magnitude of the Burgers vector together determine the radius of the hollow core. While this theory can account qualitatively for some of the properties of the nanopipe defects in $\mathrm{GaN}^{2,3}$ the ratio of the surface energy to the shear modulus is too high to account for the existence of nanopipes with diameters larger than a few $\AA$. Thus, the origin of these defects in $\mathrm{GaN}$ is an open question. In the present paper we examine the possibil- ity that adsorption of $\mathrm{H}$ or $\mathrm{NH}_{2}$ on the $\mathrm{GaN}(10 \overline{10})$ surface could reduce the surface energy, and thereby increase the equilibrium size of the nanopipe.

According to Frank, ${ }^{1}$ the equilibrium radius $r$ for a hollow-core screw dislocation is given by

$$
r=\mu b^{2} / 8 \pi^{2} \gamma,
$$

where $\mu$ is the elastic shear modulus, $b$ is the length of the Burgers vector, and $\gamma$ is the surface energy of the internal surface. This equation follows from equating the increase in surface energy, $2 \pi \gamma d r$, with the reduction in elastic energy, $\left(\mu b^{2} / 8 \pi^{2} r^{2}\right) 2 \pi r d r$, which results from increasing the radius of the hollow tube from $r$ to $r+d r$. This estimate is valid only when the tube radius is larger than the radius of the heavily reconstructed core of the dislocation. Employing $\mu=8 \times 10^{10} \mathrm{~N} / \mathrm{m}^{2}, \quad b=0.51 \mathrm{~nm}, \quad$ and $\gamma=118 \mathrm{meV} / \AA^{2}=1.89 \mathrm{~J} / \mathrm{m}^{2}$, Cherns et $^{2}{ }^{3}$ estimated the equilibrium tube diameter to be $\sim 3 \AA$. This is far less than the observed diameters, which are typically greater than $30 \AA$ even for dislocations with $b=0.51 \mathrm{~nm}$. From first-principles calculations we obtain $\mu=C_{44}=10 \times 10^{10} \mathrm{~N} / \mathrm{m}^{2}$. This value, which is in good agreement with recent experiments, ${ }^{5}$ is slightly larger than assumed by Cherns et al. and increases the expected diameter of the nanopipes by $\sim 25 \%$. More importantly, we must note that the previous estimate assumes that the appropriate surface energy is that of the bare GaN surface, which has been calculated previously ${ }^{6}$ to be $1.89 \mathrm{~J} / \mathrm{m}^{2}$. Since growth of GaN typically takes place in a hydrogen- and ammonia-rich ambient, it is possible that $\mathrm{H}$ is available to saturate the dangling bonds of the $\mathrm{Ga}$ and $\mathrm{N}$ atoms on the internal surface of the nanopipe. This could drastically reduce the formation energy of the surface and lead to larger nanopipes. We have therefore calculated the formation energy of H-terminated $\mathrm{GaN}(10 \overline{10})$ surfaces, with $\mathrm{H}$ saturating both the $\mathrm{Ga}$ and $\mathrm{N}$ dangling bonds. In addition, we have examined the structure in which the Ga dangling 
a) $\mathrm{GaN}(10 \overline{1} 0): 2 \mathrm{H}$

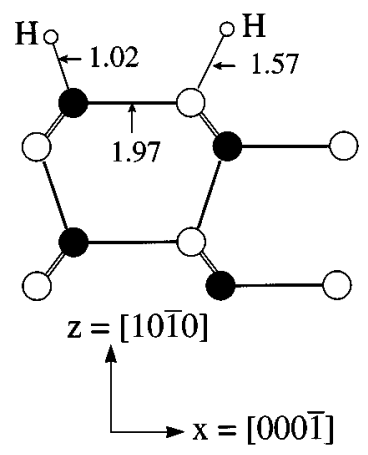

b) $\mathrm{GaN}(10 \overline{1} 0): \mathrm{NH}_{2} \mathrm{H}$

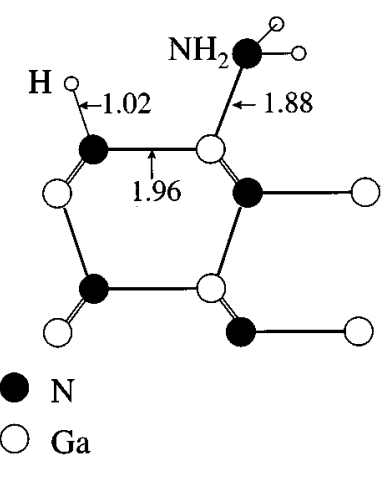

FIG. 1. (a) Schematic representation of the H-terminated $\mathrm{GaN}(10 \overline{10})$ surface. (b) $\mathrm{GaN}(10 \overline{10}): \mathrm{NH}_{2} \mathrm{H}$. Bond lengths are given in $\AA$.

bonds are saturated by $\mathrm{NH}_{2}$ and the $\mathrm{N}$ dangling bonds are saturated by $\mathrm{H}$. These structures are indicated schematically in Fig. 1.

We have performed calculations of the total energy and atomic structure within the local-density approximation and the first-principles pseudopotential approach. Forces and total energies were calculated in an iterative procedure similar to that described by Stumpf and Scheffler. ${ }^{7}$ As in previous calculations, ${ }^{6,8,9}$ soft Troullier-Martins ${ }^{10}$ pseudopotentials are employed for $\mathrm{Ga}$ and $\mathrm{N}$, and the $\mathrm{Ga} 3 d$ electrons are treated as part of the valence band. The $1 / r$ Coulomb potential is employed for $\mathrm{H}$. The plane-wave cutoff is taken to be $60 \mathrm{Ry}$ and $4 k$ points are included in the sum over the Brillouin zone. With these pseudopotentials, we calculated the structural parameters for ammonia $\left(\mathrm{NH}_{3}\right)$ and found $r(\mathrm{~N}-\mathrm{H})$ $=1.015 \AA$ and $\theta(\mathrm{H}-\mathrm{N}-\mathrm{H})=107.0^{\circ}$, in excellent agreement with the experimental values ${ }^{11} 1.012 \AA$ and $106.7^{\circ}$. For the $\mathrm{N}_{2}$ molecule we obtain a bond length of $1.075 \AA$, slightly less than the experimental value ${ }^{11}$ of $1.098 \AA$. For $\mathrm{H}_{2}$ we obtain a bond length of $0.773 \AA$, compared to the experimental value of $0.741 \AA$. The calculations indicate that the molecular reaction $\frac{1}{2} \mathrm{~N}_{2}+\frac{3}{2} \mathrm{H}_{2} \rightarrow \mathrm{NH}_{3}$ is exothermic. Including the zero-point energies of the molecules, the reaction energy is $0.8 \mathrm{eV}$, in reasonably good agreement with the experimental value, $0.4 \mathrm{eV}$. The difference between theory and experiment is most likely a consequence of the limitations of the localdensity approximation. On the basis of this comparison we expect the formation energies of the H-terminated surfaces discussed below to be accurate to within about $0.3 \mathrm{eV} /(1 \times 1$ cell $)$.

The surface energy calculations were performed for supercells containing 12 layers of $\mathrm{GaN}$ and with $\mathrm{H}$ or $\mathrm{NH}_{2}$ groups capping the $\mathrm{N}$ and $\mathrm{Ga}$ dangling bonds on each end of the GaN slab. These two structures are shown in Fig. 1. In addition to these structures and the bare surface we have performed calculations for surfaces with $\mathrm{H}$ saturating only the $\mathrm{N}$ or $\mathrm{Ga}$ atoms. The formation energy of the surface is $\Omega(T)=\frac{1}{2}\left(F-n_{\mathrm{N}} \mu_{\mathrm{N}}-n_{\mathrm{Ga}} \mu_{\mathrm{Ga}}-n_{\mathrm{H}} \mu_{\mathrm{H}}\right)$ where $F$ is the free energy of the supercell, the $n_{i}$ are the numbers of each type of atom in the cell, and the $\mu_{i}$ are the chemical potentials of the various species. The factor of 2 occurs because there are two equivalent surfaces in each unit cell. The surface energy

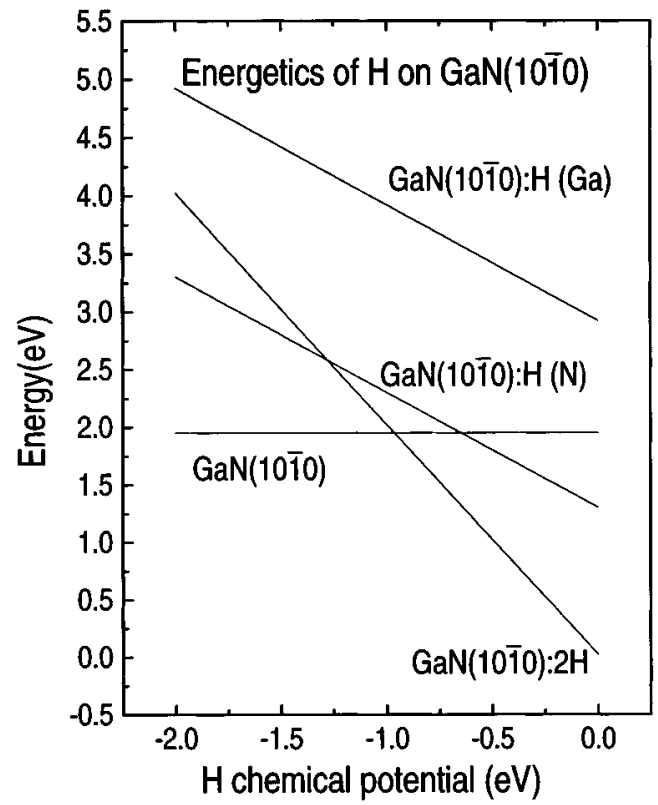

FIG. 2. Energetics of H-terminated $\mathrm{GaN}(10 \overline{10})$ surfaces as a function of the $\mathrm{H}$ chemical potential. The maximum value of $\mu_{\mathrm{H}}$ $\equiv 0.0$. The energy of the bare surface is independent of the $\mathrm{H}$ chemical potential. From these results, together with the experimental energy of the $\mathrm{H}-\mathrm{H}$ bond, $D(\mathrm{H}-\mathrm{H})=4.5 \mathrm{eV}$, it follows that the sum of the bond energies, $D(\mathrm{~N}-\mathrm{H})+D(\mathrm{Ga}-\mathrm{H})$, is approximately $6.4 \mathrm{eV}$.

$\gamma$ equals $\Omega / A$ where $A=16.424 \AA^{2}$ is the area of a $1 \times 1$ unit cell of the (1010) surface. We assume that the surface is in equilibrium with bulk $\mathrm{GaN}$; this leads to the condition $\mu_{N}+\mu_{\mathrm{Ga}}=F_{\text {bulk }}(\mathrm{GaN})$. In our approach the formation energy of the bare surface is

$$
\Omega_{\text {bare }}=\frac{1}{2}\left\{E[\mathrm{GaN}(10 \overline{10})]-12 E_{\text {bulk }}(\mathrm{GaN})\right\},
$$

where $E[\mathrm{GaN}(10 \overline{10})]$ is the energy of the 24 atom cell and $E_{\text {bulk }}(\mathrm{GaN})$ is the energy/pair of bulk GaN. We have here neglected the difference in the temperature dependence of the free energy of the slab and that of the bulk reservoir of $\mathrm{GaN}$. This results in a surface energy which is independent of temperature. The formation energy of the H-saturated surface will be approximated as

$$
\begin{aligned}
\Omega(T)= & \frac{1}{2}\{E[\mathrm{GaN}(10 \overline{10} 0): 2 \mathrm{H}] \\
& \left.-12 E_{\text {bulk }}(\mathrm{GaN})-4 \mu_{\mathrm{H}}(T)\right\}+F_{\text {ads }}(T) .
\end{aligned}
$$

Here we include explicitly the temperature dependence of the chemical potential of $\mathrm{H}$ as well as the free energy arising from the vibrational modes of the adsorbed $\mathrm{H}$. We denote this latter quantity $F_{\text {ads }}(T)$. The $\mathrm{H}$ chemical potential is bounded from above by the energy per atom in the $\mathrm{H}_{2}$ molecule. We denote this inequality $2 \mu_{\mathrm{H}} \leqslant E\left(\mathrm{H}_{2}\right)$. We have assumed that the free energy/atom arising from the vibrational degrees of freedom of the $\mathrm{Ga}$ and $\mathrm{N}$ atoms is the same for the bulk and surface. The calculations of $\mu_{\mathrm{H}}(T)$ and $F_{\text {ads }}(T)$ are discussed below.

Results for $T=0$ are shown in Fig. 2. In previous work ${ }^{6}$ the formation energy of the bare $\mathrm{GaN}(10 \overline{10})$ surface was found to be $1.95 \mathrm{eV} /(1 \times 1$ cell $)$, corresponding to $118 \mathrm{meV} / \AA^{2}$. This energy is independent of the $\mathrm{H}$ chemical 


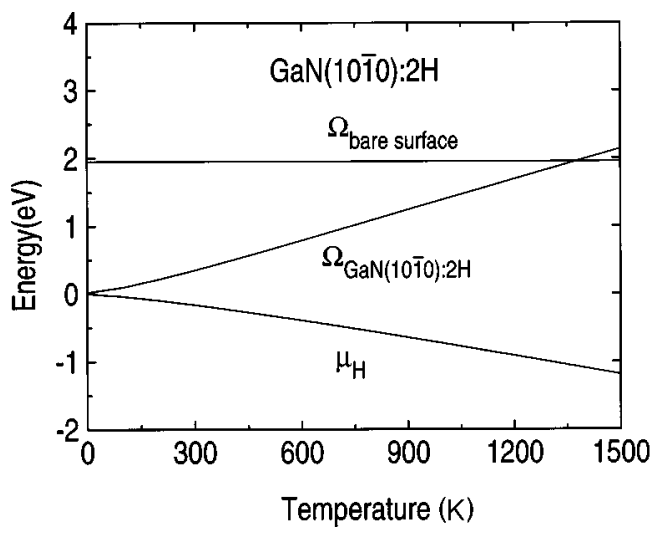

FIG. 3. Chemical potential of $\mathrm{H}$ as a function of $T$ calculated with Eq. (2). The corresponding formation energy of the $\mathrm{GaN}(1010): 2 \mathrm{H}$ surface is also shown. The nominal diameter of a nanopipe [from Eq. (1)] is $d(\AA)=7.0 / \Omega(\mathrm{eV})$ where $\Omega$ is the energy of a $1 \times 1$ unit cell of $\mathrm{GaN}(10 \overline{1} 0)$.

potential and is indicated by the horizontal line in Fig. 2. The formation energies of the surfaces in which $\frac{1}{2}$ monolayer of $\mathrm{H}$ saturates either the $\mathrm{N}$ or the $\mathrm{Ga}$ atoms are relatively high compared to the other possible structures. The origin of these high energies is clear: placing the $\mathrm{H}$ on the $\mathrm{N}$ atom results in an electron occupying a Ga-derived dangling-bond state, while placing the $\mathrm{H}$ on the $\mathrm{Ga}$ atom results in a hole in the $\mathrm{N}$-derived dangling bond. Pairing of the $\mathrm{H}$ on neighboring $\mathrm{Ga}$ and $\mathrm{N}$ atoms allows charge compensation to occur locally within a unit cell and thereby leads to a substantial energy reduction. The reaction in which two distant $\mathrm{H}$ atoms, each bonded to a $\mathrm{N}$ atom, become paired within the same unit cell is exothermic by $0.63 \mathrm{eV}$. Thus, if the $\mathrm{GaN}(10 \overline{10})$ surface is dosed with $\mathrm{H}$ atoms, we expect both $\mathrm{N}-\mathrm{H}$ and $\mathrm{Ga}-\mathrm{H}$ bonds to be formed.

The key quantity which must be known in order to determine the surface formation energy, and thereby obtain an estimate of the equilibrium radius for a $\mathrm{H}$-terminated nanopipe, is the chemical potential of $\mathrm{H}$. Using well-known results from elementary statistical mechanics ${ }^{12}$ the chemical potential of $\mathrm{H}$ is given by the expression

$$
2 \mu_{\mathrm{H}}=E\left(\mathrm{H}_{2}\right)+\tau \ln \left(p V_{Q} / \tau\right)-\tau \ln Z_{\mathrm{rot}}-\tau \ln Z_{\mathrm{vib}},
$$

where $E$ is the energy of an $\mathrm{H}_{2}$ molecule, $p$ is the pressure, $V_{Q}=\left(h^{2} / 2 \pi m k T\right)^{3 / 2}$ is the quantum volume, and $\tau=k T$ is the temperature. $Z_{\text {rot }}$ and $Z_{\text {vib }}$ are the rotational and vibrational contributions to the partition function describing the internal degrees of freedom of an $\mathrm{H}_{2}$ molecule. Metalorganic chemical-vapor deposition (MOCVD) growth of $\mathrm{GaN}$ is typically performed at a substrate temperature of order $1300 \mathrm{~K}$ and with a nominal $\mathrm{H}_{2}$ pressure of order 1 atm. At these temperatures and pressures the chemical potential of an ideal gas of $\mathrm{H}_{2}$ is much lower than $E\left(\mathrm{H}_{2}\right)$.

Shown in Fig. 3 is a plot of $\mu_{\mathrm{H}}$ as a function of $T$ for $p$ $=1 \mathrm{~atm}$. Also shown is the corresponding formation energy of the H-terminated surface. At $T=0$ the surface energy of the H-terminated $\mathrm{GaN}(10 \overline{10})$ surface is quite low: $0.02 \mathrm{eV} /(1 \times 1$ cell $)$. The energy rises to approximately $\sim 2 \mathrm{eV} /(1 \times 1$ cell $)$ at $T=1300 \mathrm{~K}$. For $T=1300 \mathrm{~K}$ the energy of the $\mathrm{H}$-saturated surface is comparable to that of the bare surface, indicating that some $\mathrm{H}$ could be present on the sur- face under growth conditions. However, since the nominal diameter of the nanopipe is $d(\AA)=7.0 / \Omega(\mathrm{eV} /$ cell $)$, nanopipes having diameters larger than a few $\AA$ are not predicted by the Frank theory for $T=1300 \mathrm{~K}$. To obtain a nanopipe of diameter $d=20 \AA$ within the Frank theory we must assume equilibration between the internal surfaces and a $\mathrm{H}$ reservoir at $T=300 \mathrm{~K}$. However, this assumption is difficult to justify on physical grounds.

Even if $\mathrm{H}$ termination is not the primary driving force for the formation of the large diameter nanopipes, it is nevertheless possible that $\mathrm{H}$ remains attached to the internal surfaces of the pipes as the sample cools following growth. To provide a characteristic signature for $\mathrm{H}$ bonded to the $\mathrm{GaN}(10 \overline{10})$ surfaces, the energies of the local vibrational modes associated with the $\mathrm{N}-\mathrm{H}$ and $\mathrm{Ga}-\mathrm{H}$ bonds have been calculated. Atomic displacements of magnitude 0.04 a.u. were employed to determine the force-constant matrix as discussed elsewhere. ${ }^{13}$ As a simplifying approximation we considered only $x$ and $z$ displacements of the $\mathrm{H}$ atoms and the surface $\mathrm{N}$ and $\mathrm{Ga}$ atoms. This allows us to obtain the bending and stretching modes associated with the N-H and $\mathrm{Ga}-\mathrm{H}$ bonds in the harmonic approximation. We find a $\mathrm{N}-\mathrm{H}$ stretching mode energy of $429 \mathrm{meV}$. Increasing the mass of the $\mathrm{N}$ atom to infinity and thereby neglecting the dynamics of the $\mathrm{N}$ atom results in a $3 \%$ reduction in the mode energy. For the Ga-H stretch energy we find $250 \mathrm{meV}$. The bending mode energies are $127 \mathrm{meV}$ for the $\mathrm{N}-\mathrm{H}$ bond and $123 \mathrm{meV}$ for the $\mathrm{Ga}-\mathrm{H}$ bond. Replacing $\mathrm{H}$ with deuterium reduces the $\mathrm{N}-\mathrm{H}$ stretching mode energy to $312 \mathrm{meV}$. The $3 \%$ difference between this value and $429 / \sqrt{2}=303 \mathrm{meV}$ arises from the component of the eigenvector on the $\mathrm{N}$ atom.

Employing these results for the vibrational energies, one may calculate the free energy of $\mathrm{GaN}(10 \overline{10} 0): 2 \mathrm{H}$ associated with the six H-related modes. Each mode of energy $E_{i}$ contributes $\Delta F_{i}=\frac{1}{2} E_{i}+\tau \ln \left[1-\exp \left(-E_{i} / \tau\right)\right]$ to the free energy $F_{\text {ads }}(T)$. One may note that $F_{\text {ads }}(T=0)$ is just the zero-point energy (ZPE) of the six H-related modes, and is equal to 0.59 eV/cell. This value may be compared with the ZPE for the $\mathrm{H}_{2}$ molecule which is $0.27 \mathrm{eV} /$ molecule. Thus, the difference in ZPE between molecular $\mathrm{H}_{2}$ and the $\mathrm{N}-\mathrm{H}$ and $\mathrm{Ga}-\mathrm{H}$ surface modes makes an important contribution to the formation energy. ${ }^{14}$ At higher temperatures $F_{\text {ads }}(T)$ decreases, and is equal to $0.40 \mathrm{eV} /$ cell at $T=1300 \mathrm{~K}$. We have included the temperature-dependent free energy of the six H-related modes in the formation energy shown in Fig. 3.

A possible kinetic pathway for the adsorption of $\mathrm{H}$ on $\mathrm{GaN}$ is through the dissociative adsorption of $\mathrm{NH}_{3}$. This could result in $\mathrm{NH}_{2}$ bonded to $\mathrm{Ga}$ and $\mathrm{H}$ bonded to $\mathrm{N}$ as shown in Fig. 1(b). For this reaction, in which the net number of $\mathrm{N}-\mathrm{H}$ bonds does not change, but one additional bond is formed between $\mathrm{Ga}$ and $\mathrm{NH}_{2}$, we find that the energy decreases by $2.08 \mathrm{eV}$. Thus, dissociative adsorption of ammonia on $\mathrm{GaN}$ is exothermic with a reaction energy of $\sim 2.08 \mathrm{eV}$. Assuming equilibrium between the surface and a vapor phase of $\mathrm{NH}_{3}$, we may write the formation energy of the surface as

$$
\begin{aligned}
\Omega= & \frac{1}{2}\left\{E\left[\mathrm{GaN}(10 \overline{1} 0): \mathrm{NH}_{2} \mathrm{H}\right]\right. \\
& \left.-12 E_{\text {bulk }}(\mathrm{GaN})-2 \mu_{\mathrm{NH} 3}(T)\right\}+F_{\text {ads }}(T) .
\end{aligned}
$$

The term $F_{\text {ads }}(T)$ depends on the vibrational degrees of 


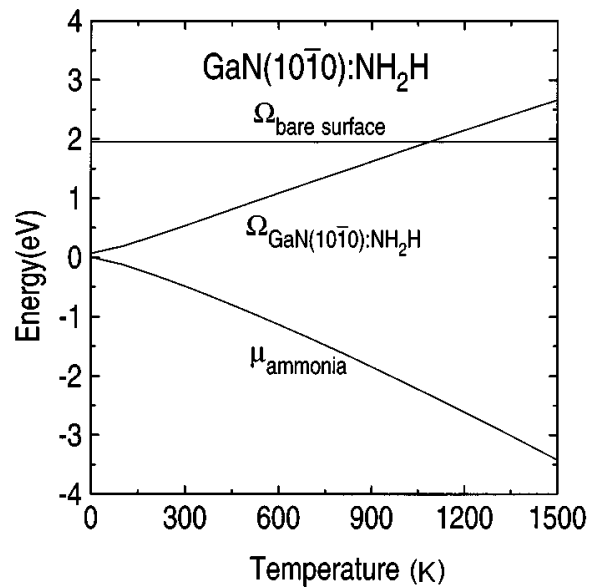

FIG. 4. Chemical potential of $\mathrm{NH}_{3}$ as a function of $T$ and the corresponding formation energy of the $\mathrm{GaN}(10 \overline{10}): \mathrm{NH}_{2} \mathrm{H}$ surface. In calculating the formation energy we have estimated the free energy of the adsorbed $\mathrm{H}$ and $\mathrm{NH}_{2}$ molecule as discussed in Ref. 15.

freedom of the adsorbed $\mathrm{H}$ and $\mathrm{NH}_{2}$ species. To estimate this quantity ${ }^{15}$ we have employed experimental values of the vibrational energies obtained in studies of $\mathrm{NH}_{2}$ adsorbed on GaAs and AlN. ${ }^{16,17}$ The formation energy of the $\mathrm{GaN}(10 \overline{10}): \mathrm{NH}_{2} \mathrm{H}$ surface is presented as a function of $T$ in Fig. 4. The surface formation energy is approximately 0.1 $\mathrm{eV} /$ cell at $T=0$, but at the growth temperature the chemical potential of $\mathrm{NH}_{3}$ is lower than its $T=0$ value by $2.9 \mathrm{eV}$, and the formation energy of the $\mathrm{NH}_{2}+\mathrm{H}$ terminated surface is correspondingly higher under such conditions.

In summary, we have examined the possibility that $\mathrm{H}$ and $\mathrm{NH}_{2}$ adsorption on the (1010) surface will reduce the surface energy and thereby enlarge the diameter of the nanopipes observed in GaN. Our calculations predict that in cases where $\mathrm{H}$ is adsorbed on the $\mathrm{GaN}(10 \overline{10})$ surface, both $\mathrm{N}-\mathrm{H}$ and $\mathrm{Ga}-\mathrm{H}$ bonds will be formed due to an attractive pairing interaction. A similar pairing interaction is expected for $\mathrm{NH}_{2}$ and $\mathrm{H}$ bonded to $\mathrm{Ga}$ and $\mathrm{N}$. To account for the minimum observed diameter (30 ̊) of nanopipe defects within Frank's model, a surface energy less than $\sim 0.2 \mathrm{eV} /(1 \times 1$ cell $)$ is required for the $(10 \overline{10})$ facet. This in turn requires that the surface equilibrates with a hydrogen reservoir having a chemical potential which satisfies the inequality $\mu_{\mathrm{H}}$ $-\frac{1}{2} E\left(\mathrm{H}_{2}\right)>\Delta$, where $\Delta \cong-0.1 \mathrm{eV}$. At the temperature and pressure believed to apply to MOCVD growth of GaN, we have $\mu_{\mathrm{H}}-\frac{1}{2} E\left(\mathrm{H}_{2}\right) \cong-1 \mathrm{eV}$. Thus, the energy of the H-terminated (1010) surface is not sufficiently low under MOCVD growth conditions to account for the large diameter nanopipes observed in experiments. While our results justify further scrutiny of the possibility ${ }^{3}$ that nanopipe formation in $\mathrm{GaN}$ is not an outcome of the equilibrium energetics of screw dislocations, we also note that we have addressed only two specific mechanisms for reducing the surface energy of the internal facets. The segregation of impurities other than $\mathrm{H}$ to the dislocation core could also play a role in the phenomenology of hollow-core screw dislocations.
${ }^{1}$ F. C. Frank, Acta Crystallogr. 4, 497 (1951).

${ }^{2}$ W. Qian, G. Rohrer, M. Skowronski, K. Doverspike, L. B. Rowland, and D. K. Gaskill, Appl. Phys. Lett. 67, 2284 (1995).

${ }^{3}$ D. Cherns, W. T. Young, J. W. Steeds, F. A. Ponce, and S. Nakamura, J. Cryst. Growth 178, 201 (1997).

${ }^{4}$ L. T. Romano (unpublished).

${ }^{5}$ A. Polian, M. Grimsditch, and I. Grzegory, J. Appl. Phys. 79, 3343 (1996).

${ }^{6}$ J. E. Northrup and J. Neugebauer, Phys. Rev. B 53, 10477 (1996).

${ }^{7}$ R. Stumpf and M. Scheffler, Comput. Phys. Commun. 79, 447 (1994).

${ }^{8}$ J. Neugebauer and C. G. Van de Walle, Phys. Rev. B 50, 8067 (1994).

${ }^{9}$ J. E. Northrup, J. Neugebauer, and L. T. Romano, Phys. Rev. Lett. 77, 103 (1996).

${ }^{10}$ N. Troullier and J. L. Martins, Phys. Rev. B 43, 1993 (1991).

${ }^{11}$ David R. Lide, Handbook of Chemistry and Physics (Chemical Rubber Company, Boca Raton, 1995).
${ }^{12}$ C. Kittel, Thermal Physics (Wiley, New York, 1969).

${ }^{13}$ J. E. Northrup, Phys. Rev. B 39, 1434 (1989).

${ }^{14}$ J. E. Northrup, Phys. Rev. B 44, 1419 (1991).

${ }^{15}$ To estimate $F_{\text {ads }}(T)$ we assume three N-H stretching modes ( $E$ $=0.43 \mathrm{eV})$, four $\mathrm{N}-\mathrm{H}$ bending modes $(E=0.13 \mathrm{eV})$, one $\mathrm{NH}_{2}$ scissors mode $(E=0.18 \mathrm{eV})$, one $\mathrm{Ga}-\mathrm{NH}_{2}$ torsion mode $(E$ $\approx 0.03 \mathrm{eV}$ ), one $\mathrm{Ga}-\mathrm{N}$ stretching mode $(E \approx 0.08 \mathrm{eV})$, and two Ga-N bending modes $(E \approx 0.05 \mathrm{eV})$. We then sum the quantity $\Delta F_{i}=\frac{1}{2} E_{i}+\tau \ln \left[1-\exp \left(-E_{i} / \tau\right)\right]$ over these 12 modes to obtain $F_{\text {ads }}(T)$. The zero-point energy from the vibrational modes of the adsorbed species is $\approx 1.1 \mathrm{eV}$. This may be compared to 0.93 $\mathrm{eV}$ for the free $\mathrm{NH}_{3}$ molecule. The estimated uncertainty in the formation energy due to the incomplete knowledge of the vibrational energies of the adsorbed $\mathrm{NH}_{2}$ molecule is $\pm 0.4 \mathrm{eV} /$ cell at $T=1300 \mathrm{~K}$.

${ }^{16}$ E. Apen and J. L. Gland, Surf. Sci. 321, 301 (1994).

${ }^{17}$ H. Liu, D. C. Bertolet, and J. W. Rogers, Jr., Surf. Sci. 320, 145 (1994). 